\title{
Caplacizumab in acquired thrombotic thrombocytopenic purpura: a profile of its use
}

\author{
Hannah A. Blair ${ }^{1} \cdot$ Katherine A. Lyseng-Williamson $^{1}$ (i)
}

Published online: 30 April 2019

(C) Springer Nature 2019, corrected publication 2019

\begin{abstract}
Caplacizumab (Cablivi $\left.{ }^{\mathrm{TM}}\right)$, an anti-von Willebrand factor Nanobody ${ }^{\circledR}$, is indicated for the treatment of adults experiencing an episode of acquired thrombotic thrombocytopenic purpura (aTTP), in conjunction with plasma exchange and immunosuppression. When combining the results of phase 2 and 3 trials in patients with aTTP, caplacizumab reduced the time to normalization of platelet count compared with placebo, and was associated with reductions in the rate of aTTP-related death, aTTP recurrence or a major thromboembolic event. Although caplacizumab was associated with more bleeding-related adverse events than placebo, most of these events were of mild or moderate severity and did not require therapeutic intervention.
\end{abstract}

Adis evaluation of caplacizumab in the treatment of aTTP

Anti-von Willebrand factor Nanobody ${ }^{\circledR}$ indicated for the treatment of aTTP, in conjunction with plasma exchange and immunosuppression

Reduces the time to normalization of platelet count

Reduces the rate of aTTP-related death, aTTP recurrence or major thromboembolic events

Reduces the need for plasma exchange and the duration of hospitalization

Associated with bleeding-related adverse events, mostly of mild to moderate severity

\section{What is the rationale for using caplacizumab in aTTP?}

Acquired thrombotic thrombocytopenic purpura (aTTP), a rare and potentially life-threatening haematological disease, is caused by reduced activity of the von Willebrand factor-cleaving metalloproteinase ADAMTS13 [1-4]. This deficiency is due to the presence of anti-ADAMTS13

Katherine A. Lyseng-Williamson

dtp@adis.com

1 Springer, Private Bag 65901, Mairangi Bay, 0754 Auckland, New Zealand autoantibodies, and causes the accumulation of ultra-large von Willebrand factor multimers in the blood, which bind to platelets and result in the formation of platelet-rich microthrombi in the microvasculature. aTTP is characterised by microangiopathic haemolytic anaemia, thrombocytopenia, ischaemia and organ damage [1-4].

Standard treatment for aTTP involves therapeutic plasma exchange in conjunction with immunosuppressive therapy (e.g. corticosteroids and rituximab) $[1,4]$. Plasma exchange is continued daily until normalization of platelet counts, resolution of haemolysis and clinical recovery [1]. Limitations of plasma exchange include complications associated with catheter placement, such as infection, bleeding and catheter thrombosis, as well as plasma infusion toxicities [3]. Patients who are refractory to plasma exchange and corticosteroids are most commonly treated with immunomodulators such as rituximab [1, 4]. However, despite these therapeutic advances, the mortality associated with aTTP remains relatively high (10-20\%), highlighting the need for novel treatments [1-3].

Caplacizumab (Cablivi ${ }^{\mathrm{TM}}$ ) has been developed to inhibit the interaction between von Willebrand factor and platelets, thereby preventing ultra-large von Willebrand factor-mediated platelet adhesion $[5,6]$. It is part of the Nanobody ${ }^{\circledR}$ platform developed by Ablynx (a Sanofi company) [7]. Nanobodies ${ }^{\circledR}$ are therapeutic proteins based on singledomain antibody fragments that contain the unique structural and functional properties of the naturally occurring heavy chain antibody [7]. 


\section{For whom is caplacizumab indicated?}

Caplacizumab, in conjunction with plasma exchange and immunosuppression, is indicated for the treatment of adults experiencing an episode of aTTP [5, 6]. Table 1 provides a summary of the prescribing information for caplacizumab in the EU [5] and USA [6].

Of note, the product approved in the EU is labelled as delivering a $10 \mathrm{mg}$ dose of caplacizumab [5], while the product approved in the USA is labelled as delivering a dose of $11 \mathrm{mg}$ [6]. This difference is due to the type of data used to establish the dose delivered by the caplacizumab kits. The dose specified in the protocol of the pivotal clinical trial (HERCULES) was $10 \mathrm{mg}$, while the dose specified in the US label was based on the results of a dose-recovery study which showed that the mean dose that could be withdrawn from the vial after reconstitution was $11 \mathrm{mg}$ [6]. Therefore, the apparent dose difference between the EU and US labels represents a regional difference in the definition of the labelled content only [8]. There is no difference in the actual dose delivered, which is equivalent to the dose used in the clinical trials [8]. In keeping with the scientific literature, hereafter, the initial and subsequent dosages of caplacizumab are referred to as $10 \mathrm{mg}$.

\section{What are the pharmacological properties of caplacizumab?}

\section{Pharmacodynamic profile}

Caplacizumab consists of two identical humanized building blocks that are genetically linked by a three-alanine linker $[5,6]$. In preclinical models of aTTP, caplacizumab inhibited the interaction between von Willebrand factor and platelet glycoprotein Ib-IV-V receptors [9, 10].

Relative to placebo in a phase 2 trial in patients with aTTP (TITAN), caplacizumab rapidly suppressed levels of von Willebrand factor-ristocetin cofactor (a biomarker for von Willebrand factor activity) activity to $<20 \%$ at day 1 ; this effect was sustained with once-daily caplacizumab throughout the 30-day treatment period [11].

Reductions in the levels of von Willebrand factor antigen and factor VIII were greater with caplacizumab than with placebo, which were assumed to be linked with an increased clearance of the caplacizumab-von Willebrand factor complex versus unbound von Willebrand factor [11]. These reductions were transient and levels returned to baseline levels within 1 week of stopping caplacizumab treatment [11].

\section{Pharmacokinetic profile}

Following intravenous administration in healthy volunteers, the pharmacokinetics of caplacizumab followed a non-linear, two-compartment model [12]. The pharmacokinetic profile of caplacizumab is dependent on the expression of the target von Willebrand factor, which is characteristic of target-mediated drug disposition $[5,6]$. Following subcutaneous administration of caplacizumab $10 \mathrm{mg}$ once daily in healthy volunteers, maximum plasma concentrations were seen $6-7 \mathrm{~h}$ post-dose, with steadystate reached following the first administration. Following subcutaneous administration in patients with aTTP, caplacizumab is rapidly and almost completely absorbed in the systemic circulation, with a central volume of distribution of $6.33 \mathrm{~L}$. The elimination half-life of caplacizumab is concentration- and target-level dependent [5, 6], with von Willebrand factor antigen levels determining the amount of caplacizumab-target complex retained in the circulation [5]. Target-bound caplacizumab is thought to undergo hepatic catabolism, while unbound caplacizumab is thought to be cleared renally $[5,6]$. The pharmacokinetics of caplacizumab are not altered to a clinically significant extent by age, gender, race, blood group or renal impairment [5, 6].

\section{What is the efficacy of caplacizumab in aTTP?}

The efficacy of caplacizumab in the treatment of aTTP has been shown in the phase 3 HERCULES [13] and phase 2 TITAN [11] trials. Patients with aTTP diagnosed on the basis of clinical presentation (i.e. presence of both thrombocytopenia and microangiopathic haemolytic anaemia with schistocytes seen on blood smear) who had received exactly one plasma-exchange treatment were eligible for participation in HERCULES [13]. In TITAN, patients were required to have an acute of aTTP with platelet count of $<100 \times 10^{9} / \mathrm{L}$, no active bleeding and a need for plasma exchange treatment [11]. The level of ADAMTS13 activity was not an inclusion or exclusion criterion. In HERCULES [13] and TITAN [11], respectively, 85 and $77 \%$ of patients had ADAMTS13 activity of $<10 \% ; 14$ and $11 \%$ had activity $\geq 10 \%$; and 1 and $12 \%$ had missing data.

Patients in these trials were randomized to receive caplacizumab or placebo in addition to standard-of-care (daily plasma exchange and immunosuppressive therapy (corticosteroids, rituximab and/or other immunosuppressants in accordance with clinical practice at each site) $[11,13]$. In HERCULES, $97 \%$ of patients received 
Table 1 Summary of the prescribing information of caplacizumab (Cablivi ${ }^{\mathrm{TM}}$ ) in acquired thrombotic thrombocytopenic purpura in the EU [5] and USA [6]. Consult local prescribing information for further details

What is the approved indication for caplacizumab?

Treatment of adults experiencing an episode of aTTP, in conjunction with plasma exchange and immunosuppression

How is caplacizumab available?

Single-dose vial containing $10(\mathrm{EU})$ or $11 \mathrm{mg}(\mathrm{USA})^{\mathrm{a}}$ of caplacizumab powder for injection

How should caplacizumab be stored, prepared and administered?

Storage and preparation Ensure vial and pre-filled syringe are at room temperature; reconstitute and dilute powder prior to injection; store reconstituted solution in the refrigerator $\left(2-8{ }^{\circ} \mathrm{C}\right)$; administer within $4 \mathrm{~h}$ of reconstitution

Method of administration IV: administer as a bolus injection

SC: inject into a site on the abdomen; avoid area around the navel; rotate injection sites

At what dosage and for how long should caplacizumab be used?

Initial IV injection $\quad 10(\mathrm{EU})$ or $11 \mathrm{mg}(\mathrm{USA})^{\mathrm{a}}$ prior to plasma exchange on the first day of treatment

Subsequent SC injections $10(\mathrm{EU})$ or $11 \mathrm{mg}(\mathrm{USA})^{\mathrm{a}}$ following each plasma exchange for the duration of daily plasma exchange treatment, and for 30 days after stopping plasma exchange treatment

Evidence of unresolved Optimize the immunosuppression regimen and continue daily SC administration of caplacizumab until the signs immunological disease at the end of the 30-day period (EU)

Signs of persistent under- Extend treatment for a maximum of 28 days lying disease after initial treatment course (USA)

What are the contraindications to the use of caplacizumab?

Hypersensitivity to the active substance/excipients

How should caplacizumab be used in special populations?

Paediatric patients Efficacy and safety have not been established

Elderly patients No special precautions or dosage adjustments are needed (EU)

Patients with renal impair- No dosage adjustment is necessary (EU)

ment

Patients with hepatic No dosage adjustment is necessary (EU)

impairment Severe impairment: conduct benefit/risk assessment and closely monitor patients (EU); closely monitor patients for bleeding (USA)

Pregnant women Preferable to avoid use during pregnancy (EU); no available data regarding its use in pregnancy (USA)

Breastfeeding women Consider the benefits of breastfeeding to the infant together with the mother's clinical need for caplacizumab (EU,

USA); consider any potential adverse effects to the infant from the drug or underlying maternal condition (USA)

What special warnings/precautions pertain to the use of caplacizumab?

Active clinically signifi- Interrupt treatment (EU, USA); consider use of von Willebrand factor concentrate to correct haemostasis (EU, cant bleeding USA); closely monitor patients for signs of bleeding if treatment is restarted (USA)

How should the increased risk of bleeding with caplacizumab be managed?

Concomitant anticoagu- Conduct benefit/risk assessment (EU); closely monitor patients (EU, USA)

lants or antiplatelets

In patients with underlying Closely monitor patients (EU)

coagulopathies

In patients undergoing Withhold treatment for 7 days prior to elective surgery, dental procedures or other invasive interventions; consider surgery use of von Willebrand factor concentrate if emergency surgery is needed

aTTP acquired thrombotic thrombocytopenic purpura, $I V$ intravenous, $S C$ subcutaneous

${ }^{a}$ Doses are equivalent. The slightly higher nominal dose in the USA is based on the results of a dose-recovery study, which showed that the mean dose that could be withdrawn from the vial after reconstitution was $11 \mathrm{mg}$, rather than the $10 \mathrm{mg}$ dose specified in HERCULES [6]

corticosteroids; $17 \%$ received rituximab started by trial day 3 and $12 \%$ received rituximab stated after trial day 3 [13]. During daily plasma exchange in TITAN, $91 \%$ of patients received corticosteroids and $15 \%$ received rituximab [11].
In both trials, subcutaneous administration of caplacizumab was preceded by an intravenous loading dose to ensure rapid attainment of concentrations required for von Willebrand factor inhibition [11]. In HERCULES, patients received an intravenous loading dose of caplacizumab $10 \mathrm{mg}$ 
or placebo prior to the start of the first plasma exchange after randomization, then subcutaneous caplacizumab $10 \mathrm{mg}$ or placebo was administered once daily until 30 days after the last plasma exchange [13]. Treatment could be extended for a maximum of 28 days, according to risk factors for aTTP recurrence (e.g. persistent severe ADAMTS13 deficiency) [13]. In TITAN, patients received an intravenous loading dose of caplacizumab $10 \mathrm{mg}$ or placebo anytime from $6 \mathrm{~h}$ before to $15 \mathrm{~min}$ before the start of the first plasma exchange after study enrolment [11]. Once-daily subcutaneous caplacizumab $10 \mathrm{mg}$ or placebo was administered within $30 \mathrm{~min}$ after the end of each plasma exchange, and continued for 30 days after the last plasma exchange. The maximum duration of treatment was 90 days [11].

\section{HERCULES trial}

The median time to normalization of the platelet count was significantly shorter in the caplacizumab group than in the placebo group in the HERCULES trial (Table 2) [13]. Caplacizumab recipients were 1.55 times more likely than placebo recipients to achieve normalization of the platelet count. Caplacizumab was associated with a significant reduction in the incidence of the composite endpoint of aTTP-related death, aTTP recurrence or a major thromboembolic event during the trial treatment period. During the overall trial period (including the 28-day follow-up period), the incidence of aTTP recurrence was also significantly lower with caplacizumab than with placebo (Table 2). All recurrences in the placebo group occurred within 30 days after the end of daily plasma exchange, meeting the criteria for exacerbations. Conversely, most recurrences in the caplacizumab group occurred during the 28-day followup period, meeting the criteria for relapse. All relapses in the caplacizumab group occurred in patients with suppressed ADAMTS13 activity (i.e. $<10 \%$ ), indicative of the presence of unresolved underlying autoimmune disease [13].

Compared with placebo, caplacizumab recipients experienced clinically meaningful reductions in plasma exchange use, volume of plasma exchanged, duration of hospitalization and length of stay in the ICU (Table 2) [13]. Among patients who achieved normalization of the platelet count $(n=129), 60 \%$ of caplacizumab recipients and $53 \%$ of placebo recipients continued to have severely suppressed (i.e. $<10 \%)$ ADAMTS13 activity in the week after stopping daily plasma exchange [13].

\section{TITAN trial}

In the overall population $(n=75)$, caplacizumab significantly reduced the time to response (i.e. time to normalization of the platelet count) by $39 \%$ compared with placebo in the TITAN trial (Table 2) $[11,14]$. In a post hoc analysis of patients with baseline ADAMTS13 activity of $<10 \%$ $(n=58)$, the median time to response was 3.0 days with caplacizumab and 4.6 days with placebo (event rate ratio 1.63; 95\% CI 0.92-2.92) [11]. The rate of complete remission after the initial course of daily plasma exchange was higher with caplacizumab than with placebo, while rates of exacerbation were lower with caplacizumab than with placebo. In 7 of 8 caplacizumab recipients who experienced a relapse during the 1-month follow-up period, the recurrence occurred within 10 days of stopping the drug. In these patients, ADAMTS13 activity levels were persistently suppressed (i.e. $<10 \%$ ). The mean number of plasma exchange days and the mean volume of plasma exchanged were lower in the caplacizumab group than in the placebo group (Table 2) [11].

According to a post hoc analysis, caplacizumab reduced the frequency of major thromboembolic events, aTTP exacerbations and aTTP-related mortality compared with placebo (Table 2) [15]. Overall, $11 \%$ of caplacizumab and $43 \%$ of placebo recipients experienced one or more thromboembolic events, experienced an exacerbation, or died. With the exception of three events in the placebo group, all events occurred in patients with a baseline ADAMTS13 activity of $<10 \%$. Most events occurred during the first 30 days of the trial, with most thromboembolic events occurring during the daily plasma exchange period [15].

\section{Integrated analysis}

An integrated efficacy analysis of HERCULES and TITAN $(n=220)$ confirmed the results of the individual trials, demonstrating that caplacizumab significantly $(p=0.0006)$ reduced the time to platelet count response versus placebo (platelet count normalization rate ratio 1.65 ; 95\% CI 1.24-2.20) [16]. Compared with placebo, caplacizumab was associated with clinically meaningful and statistically significant reductions in the rate of aTTP-related death, aTTP recurrence (exacerbation) or a major thromboembolic event; the rate of aTTP recurrence during the entire study period; and the proportion of patients with refractory aTTP (Table 2). The mortality rate was significantly lower with caplacizumab than with placebo during the double-blind/ single-blind treatment period ( 0 vs $4 \% ; p=0.0477$ ) but not during the entire study period (1 vs $5 \%$ ) [16].

\section{What is the tolerability profile of caplacizumab?}

In an integrated safety analysis of pooled data from TITAN and HERCULES $(n=220)$, treatment-emergent adverse events (TEAEs) were reported in $96.2 \%$ of caplacizumab 
Table 2 Efficacy of caplacizumab in conjunction with plasma exchange and immunosuppressive therapy in patients with an acute episode of acquired thrombotic thrombocytopenic purpura in randomized trials

Comparators Key results (caplacizumab vs placebo)

\section{HERCULES (double-blind phase 3 trial) [13]}

Caplacizumab $10 \mathrm{mg} \quad$ Median time to normalization of platelet count ${ }^{\mathrm{a}}$ (days): 2.69 vs 2.88 (RR 1.55; 95\% CI 1.09-2.19)**

once daily $(n=72) \quad$ Composite outcome of aTTP-related death, aTTP recurrence ${ }^{\mathrm{b}}$ or major TEE (\% pts): 12 vs $49 * * *$

vs placebo $(n=73) \quad$ aTTP recurrence ${ }^{\mathrm{b}}$ during overall trial period (\% pts): 12 vs $38^{* * *}$

Refractory aTTP ${ }^{\mathrm{c}}$ (\% pts): 0 vs 4

Median time to normalization of organ-damage markers (days): 2.86 vs 3.36

Mean no. of days of plasma exchange: 5.8 vs 9.4

Mean volume of plasma exchanged (L): 21.3 vs 35.9

Mean duration of hospitalization (days): 9.9 vs 14.4

ICU admission (\% pts): 39 vs 37

Mean no. of days in ICU: 3.4 vs 9.7

TITAN (single-blind phase 2 trial) [11, 14]

Caplacizumab $10 \mathrm{mg}$ Median time to platelet count response ${ }^{\mathrm{d}}$ (days): 2.97 vs 4.79 (RR 2.20; 95\% CI 1.28-3.78)**

once daily $(n=36)$

vs placebo $(n=39)$

Complete remission after initial course of daily plasma exchange $\left.\mathrm{e}^{\mathrm{e}} \% \mathrm{pts}\right): 81 \mathrm{vs} 46$

aTTP exacerbation $^{\mathrm{f}}$ (\% pts): 8 vs 28

aTTP relapse ${ }^{\mathrm{g}}$ during 1-month follow-up period (\% pts): 22 vs 0

aTTP relapse ${ }^{\mathrm{g}}$ during 12-month follow-up period (\% pts): 31 vs 8

Mean no. of days of plasma exchange: 7.7 vs 11.7

Mean volume of plasma exchanged (L): 25.8 vs 41.8

Post hoc analysis [15] Major TEE (\% pts): 3 vs 19

aTTP-related mortality (\% pts): 0 vs 5

\section{Integrated analysis of HERCULES and TITAN [16]}

Caplacizumab $10 \mathrm{mg} \quad$ aTTP-related death, aTTP recurrence ${ }^{\mathrm{b}}$ or major TEE (\% pts): 13 vs $47 * * *$

once daily $(n=108) \quad$ aTTP recurrence ${ }^{\mathrm{b}}$ during entire study period (\% pts): 18 vs $35^{* *}$

vs placebo $(n=112)$

Refractory aTTP ${ }^{\mathrm{c}}\left(\%\right.$ pts): 0 vs $6^{* *}$

Mortality (\% pts): 0 vs $4 *$

Mean no. of days of plasma exchange: 6.5 vs 10.4

Unless otherwise stated, all secondary outcomes were assessed during the trial treatment period

aTTP acquired thrombotic thrombocytopenic purpura, $I C U$ intensive care unit, $L D H$ lactate dehydrogenase, pts patients, $R R$ rate ratio, $T E E$ thromboembolic event

$* p<0.05, * * p \leq 0.01, * * * p<0.001$

a Primary endpoint (defined as an initial platelet count $\geq 150 \times 10^{9} / \mathrm{L}$ with subsequent discontinuation of daily plasma exchange within 5 days)

${ }^{b}$ New decrease in platelet count necessitating reinitiation of plasma exchange after initial platelet count normalization

${ }^{c}$ Lack of doubling of platelet count after 4 days of treatment and LDH level above upper limit of normal

${ }^{\mathrm{d}}$ Primary endpoint (defined as a platelet count $\geq 150 \times 10^{9} / \mathrm{L}$, confirmed at $48 \mathrm{~h}$ with LDH level no more than twice upper limit of normal)

${ }^{\mathrm{e}}$ Confirmed normalization of platelet count and absence of exacerbations

${ }^{\mathrm{f}}$ Episode of thrombocytopenia occurring 1-30 days after last daily plasma exchange session requiring reinitiation of daily plasma exchange

${ }^{\mathrm{g}} \mathrm{New}$ episode of thrombocytopenia occurring $>30$ days after the last daily plasma exchange session

recipients and $95.5 \%$ of placebo recipients [17]. TEAEs that were significantly more common ( $\geq 5 \%$ difference) with caplacizumab than with placebo were epistaxis ( 29 vs $6 \%$ ), headache (21 vs $14 \%$ ) and gingival bleeding (16 vs 3\%). Most of these adverse events were of mild severity [17]. Other adverse reactions commonly observed with caplacizumab ( $\geq 10 \%$ incidence) include pyrexia, fatigue and urticaria [5]. The proportion of patients experiencing serious adverse events was $29 \%$ in the caplacizumab group and $49 \%$ in the placebo group [17]. Seven percent of caplacizumab recipients and $10 \%$ of placebo recipients discontinued treatment due to adverse events [17].

A post hoc safety analysis of HERCULES demonstrated that after normalization for time of exposure, the incidence rate per 100 patients months of any TEAE was 535.6 with caplacizumab and 821.7 with placebo [18]. TEAEs occurring at a higher incidence with caplacizumab than with placebo were epistaxis (39.1 vs 3.3 per 100 patients months) 
and gingival bleeding (14.9 vs 1.7 per 100 patients months). The incidence rate per 100 patients months of any serious adverse event was 26.4 with caplacizumab and 83.3 with placebo [18].

Caplacizumab increases the risk of bleeding [6]. The risk of bleeding is increased in patients receiving concomitant anticoagulants or antiplatelets, in patients with underlying coagulopathies (e.g. haemophilia and other coagulation factor deficiencies) and in patients undergoing surgery (Table 1) [5, 6]. In HERCULES, bleeding-related TEAEs were reported in $62 \%$ of caplacizumab recipients and $47 \%$ of placebo recipients [19]. The most common ( $\geq 5 \%$ incidence) bleeding-related TEAEs were epistaxis (27 vs $3 \%$ ), gingival bleeding (17 vs $1 \%$ ), contusion (7 vs $14 \%$ ) and catheter site haemorrhage (7 vs 7\%). The majority of bleeding-related TEAEs were of mild or moderate severity, and most did not require therapeutic intervention [19]. In clinical trials, bleeding events with caplacizumab occurred in different body systems and this was independent of duration of treatment; although some of these bleeding events were serious and required medical attention, most were self-limited and all resolved [5]. Caplacizumab should be interrupted in patients with active clinically significant bleeding, and the use of von Willebrand factor concentrate should be considered (Table 1) [5, 6].

\section{What is the current clinical position of caplacizumab in aTTP?}

Caplacizumab represents an effective treatment option for adults experiencing an episode of aTTP. In conjunction with plasma exchange and immunosuppressive therapy, caplacizumab reduces the time to normalization of

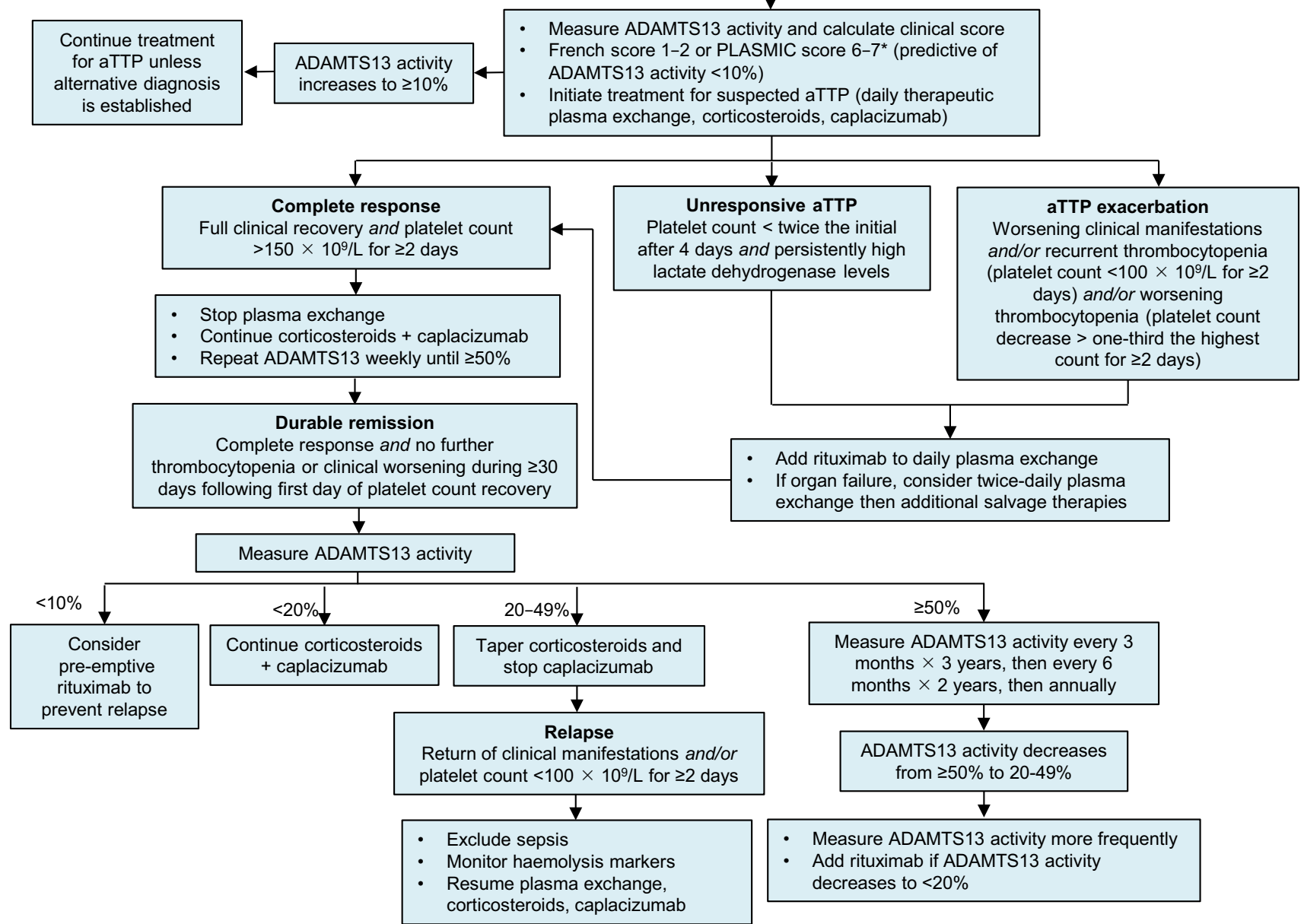

Fig. 1 Suggested evaluation and therapeutic management of acquired thrombotic thrombocytopenic purpura (aTTP), based on recommendations by Coppo et al. [3] and Joly et al. [1]. Evaluation and manage- ment protocols may differ in other healthcare centres. *If intermediate likelihood for ADAMTS13 activity < 10\% (i.e. PLASMIC score of 5), empiric treatment for aTTP is recommended 
platelet count compared with placebo. Caplacizumab is also associated with reductions in the rate of aTTP-related death, aTTP recurrence or a major thromboembolic event. Importantly, caplacizumab reduces the number of days of plasma exchange, volume of plasma exchanged, duration of hospitalization and length of stay in the intensive care unit. Although caplacizumab was associated with more bleeding-related adverse events than placebo, most of these events were of mild or moderate severity and did not require therapeutic intervention.

Two clinical scores (the French score and the PLASMIC score) are used to identify patients with severe ADAMTS13 deficiency (Fig. 1) [3]. Both scoring systems use the absence of an associated condition (e.g. cancer, transplant), severe thrombocytopenia (platelet count $<30 \times 10^{9} / \mathrm{L}$ ) and mild renal involvement (serum creatinine level of $<2$ or $2.25 \mathrm{mg}$ / dL) as criteria for identifying patients with probable aTTP [3]. Daily therapeutic plasma exchange, with or without corticosteroids, is recommended for the first-line treatment of aTTP (Fig. 1) [1]. In patients who fail to achieve a complete response, or who experience aTTP exacerbation, second-line therapy with plasma exchange + rituximab often induces complete remission [1]. Caplacizumab may also be used for the initial treatment of aTTP (Fig. 1) [3].

Well-designed trials directly comparing caplacizumab with other aTTP therapies would help to clarify its position in the management of aTTP. Other novel agents currently being investigated for the treatment of aTTP include recombinant ADAMTS13 (rADAMTS13), $N$-acetylcysteine, anfibatide and bortezomib [4]. Evidence suggests that combining rADAMTS 13 with immunosuppressive therapy or caplacizumab may reduce or possibly eliminate the need for plasma exchange. Because caplacizumab does not affect the autoimmune pathobiology of aTTP, it will always need to be used in combination with immunosuppressive therapy [4].

In addition to data evaluating the efficacy and tolerability of caplacizumab relative to other aTTP therapies in headto-head clinical trials, longer-term efficacy, tolerability and cost-utility data for caplacizumab are needed. An open-label, phase 3, prospective follow-up trial (post-HERCULES) is currently underway to evaluate the long-term safety and efficacy of caplacizumab in patients with aTTP who have completed the HERCULES trial (NCT02878603). Results from this trial are expected in late 2020 and are awaited with interest.

Acknowledgements The manuscript was reviewed by: A. Antonelli, Department of Clinical and Experimental Medicine, University of Pisa, Pisa, Italy; W. F. Clark, Division of Nephrology, London Health Sciences Centre, London, ON, Canada; J. de la Rubia, Department of Hematology, University Catolica Valencia Hospital, Valencia, Spain; P. Knoebl, Division of Hematology and Hemostasis, Department of Medicine 1, Medical University of Vienna, Vienna, Austria; L. Rice, Department of Medicine, Houston Methodist Hospital, Houston, TX,
USA; G. Spectre, Institute of Hematology, Rabin Medical Center, Bellison Hospital, Petah-Tikva and Sackler School of Medicine, Tel Aviv University, Tel Aviv, Israel. During the peer review process, Ablynx (a Sanofi company), the marketing-authorization holder of caplacizumab in the EU, was also offered an opportunity to provide a scientific accuracy review of their data. Changes resulting from comments received were made on the basis of scientific and editorial merit.

\section{Compliance with ethical standards}

Funding The preparation of this review was not supported by any external funding.

Conflict of interest H. A. Blair and K. A. Lyseng-Williamson are employees of Adis/Springer, are responsible for the article content and declare no conflicts of interest.

Open Access This article is distributed under the terms of the Creative Commons Attribution-NonCommercial 4.0 International License (http://creativecommons.org/licenses/by-nc/4.0/), which permits any noncommercial use, duplication, adaptation, distribution and reproduction in any medium or format, as long as you give appropriate credit to the original author(s) and the source, provide a link to the Creative Commons license and indicate if changes were made.

\section{References}

1. Joly BS, Coppo P, Veyradier A. Thrombotic thrombocytopenic purpura. Blood. 2017;129(21):2836-46.

2. Von Veyradier A. Willebrand factor: a new target for TTP treatment? N Engl J Med. 2016;374(6):583-5.

3. Coppo P, Cuker A, George JN. Thrombotic thrombocytopenic purpura: toward targeted therapy and precision medicine. Res Pract Thromb Haemost. 2018. https://doi.org/10.1002/rth2.12160.

4. Masias C, Cataland SR. Novel therapies in thrombotic thrombocytopenic purpura. Res Pract Thromb Haemost. 2018;2(1):19-26.

5. Cablivi (caplacizumab $10 \mathrm{mg}$ powder and solvent for solution for injection): EU summary of product characteristics. Zwijnaarde (Belgium): Ablynx NV; 2018.

6. Cablivi ${ }^{\circledR}$ (caplacizumab-yhdp) for injection, for intravenous or subcutaneous use: US prescribing information. Cambridge (MA): Genzyme Corporation; 2019.

7. Ablynx. Understanding nanobodies; 2018. http://www.ablyn x.com/technology-innovation/understanding-nanobodies. Accessed 13 Mar 2019.

8. Data on file, Sanofi, 2019.

9. Callewaert F, Roodt J, Ulrichts H, et al. Evaluation of efficacy and safety of the anti-VWF Nanobody ALX-0681 in a preclinical baboon model of acquired thrombotic thrombocytopenic purpura. Blood. 2012;120(17):3603-10.

10. Ulrichts H, Silence K, Schoolmeester A, et al. Antithrombotic drug candidate ALX-0081 shows superior preclinical efficacy and safety compared with currently marketed antiplatelet drugs. Blood. 2011;118(3):757-65.

11. Peyvandi F, Scully M, Kremer Hovinga JA, et al. Caplacizumab for acquired thrombotic thrombocytopenic purpura. N Engl J Med. 2016;374(6):511-22.

12. Klamroth R, Holz JB, Vercruysse K, et al. Results from a healthy volunteer, single-dose, phase 1 study investigating the safety, tolerability, and pharmacokinetics of the nanobody ${ }^{\mathrm{TM}}$ ALX-0081 
targeting von Willebrand factor [abstract no. P212]. Arterioscler Thromb Vasc Biol. 2008;28(6):e72.

13. Scully M, Cataland SR, Peyvandi F, et al. Caplacizumab treatment for acquired thrombotic thrombocytopenic purpura. N Engl J Med. 2019. https://doi.org/10.1056/nejmoa1806311.

14. Cablivi (caplacizumab): EU assessment report. London: European Medicines Agency; 2018.

15. Peyvandi F, Scully M, Kremer Hovinga JA, et al. Caplacizumab reduces the frequency of major thromboembolic events, exacerbations and death in patients with acquired thrombotic thrombocytopenic purpura. J Thromb Haemost. 2017;15(7):1448-52.

16. Peyvandi F, Cataland SR, Scully M, et al. Integrated efficacy results from the phase II and phase III studies with caplacizumab in patients with acquired thrombotic thrombocytopenic purpura. Blood. 2018;132(Suppl 1):373.
17. Knöbl P, Scully M, Cataland SR, et al. Integrated safety results from the phase II and phase III studies with caplacizumab in patients with acquired thrombotic thrombocytopenic purpura. Blood. 2018;132(Suppl 1):3739.

18. Kremer Hovinga JA, Scully M, Cataland SR, et al. Safety of caplacizumab for the treatment of patients with acquired thrombotic thrombocytopenic purpura: results normalized to time of exposure in a double-blind, placebo-controlled, phase 3 Hercules study [abstract]. Blood. 2018;132(Suppl 1):3744.

19. Cataland SR, Scully M, Peyvandi F, et al. Risk factors and manageability of the mainly mild mucocutaneous bleeding profile observed in aTTP patients treated with caplacizumab during the phase III Hercules study [abstract]. Blood. 2018;132(Suppl 1): 1142 . 